\title{
INTEGRABILITY OF TRIGONOMETRIC SERIES
}

\section{S. ZAHID ALI ZENEI}

\begin{abstract}
A bstract. Generalization of the theorems of Taljakovskii [7] and Sing and Sharma [5] have been obtained.
\end{abstract}

1. A sequence $\left\langle a_{n}\right\rangle$ of positive numbers is said to be quasi-monotone if $\Delta a_{n} \geq-\propto$ $a_{n} / n$ for some positive $\propto$. It is obvious that every null monotonic decreasing sequence is quasi-monotone. A sequence $\left\langle a_{n}\right\rangle$ is said to be $\delta$-quasi-monotone if $\left.a_{n} \rightarrow 0, a_{n}\right\rangle 0$ ultimately and $\Delta a_{n} \geq-\delta_{n}$, where $\left\langle\delta_{n}\right\rangle$ is a sequence of positive numbers. Clearly a null quasi monotone sequence is $\delta$-quasi-monotone with $\delta_{n}=\propto a_{n} / n$.

We say that a sequence $\left\langle a_{n}>\right.$ of numbers satisfies condition $S$ or $a_{n} \in S$, if $a_{n} \rightarrow 0$ as $n \rightarrow \infty$ and there exists a sequence of numbers $\left\langle A_{k}\right\rangle$ such that
(a) $A_{k} \downarrow 0$
(b) $\Sigma_{k=1}^{\infty} A_{k}<\infty$, and

(c) $\left|\Delta a_{k}\right| \leq A_{k}$, for all $k$.

By replacing the condition (a) of (1.1) only by:

$\left(\mathrm{a}^{\prime}\right)<A_{k}>$ is quasi-monotone

$\left(\mathrm{a}^{\prime \prime}\right)<A_{k}>$ is $\delta$-quasi-monotone and $\Sigma k \delta_{k}<\infty$, we say that $<a_{n}>\in S(\propto)$ and $<a_{n}>\in S(\delta)$ respectively.

Thus, in view of the above definitions it is obvious that $S \subset S(\propto) \subset S(\delta)$. And all these three are the generalization of quasi-convex sequence. Our condition $S(\delta)$ is weaker than the conditions $S$ and $S(\propto)$ of Sidon [4] and that of Sing and Sharma [5] respectively.

2. Let

$$
f(x)=\frac{1}{2} a_{0}+\sum_{n=1}^{\infty} a_{n} \cos n x
$$

and

$$
g(x)=\sum_{n=1}^{\infty} a_{n} \sin n x,
$$

Received January 9, 1989, reviewed February 12, 1990. 
be the trigonometric series.

Integrability of the above series has been discussed by several authors, for example Young [8], Kolmogorov [3] and Sidon [4]. In 1973, Teljakoveskii [7] has proved the following Theorems by taking a set of weaker conditions of Sidon [4] than those of the earlier authors.

Theorem A. Let the coefficient of the series $f(x)$ satisfy the condition $S$. Then the series is a Fourier series and the following relation holds

$$
\int_{0}^{\pi}|f(x)| d x \leq C \sum_{n=0}^{\infty} A_{n}
$$

where $C$ is an absolute constant.

Theorem $\mathbb{B}$. Let the coefficient of series $g(x)$ satisfy the condition $S$. Then the following relation holds for $p=1,2, \ldots$

$$
\int_{\pi / p+1}^{\pi}|g(x)| d x \leq \sum_{n=1}^{p} \frac{\left|a_{n}\right|}{n}+O\left(\sum_{n=1}^{\infty} A_{n}\right)
$$

In particular $g(x)$ is a Fourier series iff $\sum_{n=1}^{\infty} \frac{a_{n}}{n}<\infty$.

Very recently Sing and Sharma [5] have proved Theorem A and B for the class $S(\propto)$. In this paper we generalize Theorem $A$ and $B$ for the class $S(\delta)$, so as to get the above mentioned generalization of Taljakoveskii [7] and Sing and Sharma [5] as a special cases.

3. We prove the following theorem.

Theorem 1. Let the coefficient of the series $f(x)$ satisfy the condition $S(\delta)$. Then the series is a Foureir series and the following relation holds

$$
\int_{0}^{\pi}\left|\frac{a_{0}}{2}+\sum_{n=1}^{\infty} a_{n} \cos n x\right| d x \leq C \sum_{n=0}^{\infty} A_{n}
$$

where $C$ is an absolute constant

Theorem 2. Let the coefficient of series $g(x)$ satisfy the condition $S(\delta)$. Then the series coverges to a function and the following relation holds for $p=1,2,3 \ldots$

$$
\int_{\pi / p+1}^{\pi}\left|\sum_{n=1}^{\infty} a_{n} \sin n x\right| d x \leq \sum_{n=1}^{p} \frac{\left|a_{n}\right|}{n}+O\left(\sum_{n=1}^{\infty} A_{n}\right) .
$$

4. For the proof of the above theorem we require the following lemmas. 
Lemma $1[2]$. If the sequence of numbers $\langle\propto\rangle$ satisfies the condition $\left|\propto_{i}\right| \leq 1$, then

$$
\int_{0}^{\pi}\left|\sum_{i=0}^{k} \propto_{i} \frac{\sin (i+1 / 2) x}{2 \sin x / 2}\right| d x \leq C(k+1)
$$

and

$$
\int_{\pi / p+1}^{\pi}\left|\sum_{i=0}^{k} \propto_{i} \frac{\cos (i+1 / 2) x}{2 \sin x / 2}\right| d x \leq C(k+1),
$$

Where $C$ is a positive absolute constant.

Lemma 2 [1]. If $\left\langle a_{n}\right\rangle$ is $\delta$-quasi-monotone with $\Sigma n^{v} \delta_{n}<\infty v \neq 0$ then the convergence of $\Sigma n^{v-1} a_{n}$ implies that $n^{v} a_{n} \rightarrow 0, n \rightarrow \infty$.

Lemma 3. Let $\left\langle a_{n}\right\rangle$ be a $\delta$-quasi-monotone sequence with

$$
\sum_{n=1}^{\infty} n \delta_{n}<\infty \text {. If } \sum_{n=1}^{\infty} a_{n}<\infty \text {, then } \sum_{n=1}^{\infty}(n+1)\left|\Delta a_{n}\right|<\infty \text {. }
$$

Proof. By partial summation we have

$$
\sum_{k=1}^{n} a_{k}=\sum_{k=1}^{n-1}(k+1) \Delta a_{k}+(n+1) a_{n}-a_{1}
$$

Since $\left\langle a_{n}\right\rangle$ is $\delta$-quasi-monotone sequence and $\Sigma_{k=1}^{\infty} a_{k}<\infty$, we have $n a_{n}=o(1)$, by Lemma 2. Therefore, by taking the limit we have,

$$
\sum_{k=1}^{\infty} a_{k}=\sum_{k=1}^{\infty}(k+1) \Delta a_{k}-a_{1}
$$

From which it is clear that $\Sigma_{k=1}^{\infty}(k+1) \Delta a_{k}<\infty$.

Now,

$$
\begin{aligned}
\sum_{k=1}^{\infty}(k+1)\left|\Delta a_{k}\right| & =\sum_{k=1}^{\infty}(k+1)\left|a_{k}-a_{k+1}+\delta_{k}-\delta_{k}\right| \\
& \leq \sum_{k=1}^{\infty}(k+1)\left(a_{k}-a_{k+1}+\delta_{k}\right)+\sum_{k=1}^{\infty} \delta_{k}(k+1) \\
& =\sum_{k=1}^{\infty}(k+1) \Delta a_{k}+2 \sum_{k=1}^{\infty} \delta_{k}(k+1) \\
& <\infty,
\end{aligned}
$$


by virtue of the hypothesis.

5. Proof of Theorem 1. By virtue of hypothesis $\Delta a_{n} \geq-\delta_{n}$, we have

$$
\left|\Delta a_{n}\right| \leq \Delta a_{n}+2 \delta_{n}
$$

Also the convergence of $\sum_{k=1}^{\infty} k \delta_{k}$ implies that $\Sigma_{k=1}^{\infty} \delta_{k}<\infty$. Therefore, using the condition that $a_{n} \rightarrow 0$, we have

$$
\sum_{n=1}^{\infty}\left|\Delta a_{n}\right| \leq \sum_{n=1}^{\infty} \Delta a_{n}+2 \sum_{n=1}^{\infty} \delta_{n}<\infty .
$$

Thus $\frac{a_{0}}{2}+\sum_{n=1}^{\infty} a_{n} \cos n x$ converges to $f(x)$ for all $x$ except possibly $x=0$.

By summation by parts, we have

$$
\begin{aligned}
f(x) & =\lim _{n \rightarrow \infty}\left[\frac{a_{0}}{2}+\sum_{k=1}^{n} a_{k} \cos k x\right] \\
& =\lim _{n \rightarrow \infty}\left[\frac{a_{0}}{2}+\sum_{k=1}^{n-1} D_{k}(x) \Delta a_{k}+a_{n} D_{n}(x)-\frac{a_{0}}{2}\right] \\
& =\lim _{n \rightarrow \infty}\left[\sum_{k=1}^{n-1} D_{k}(x) \Delta a_{k}+a_{n} D_{n}(x)\right] \\
& =\sum_{k=1}^{\infty} \Delta a_{k} D_{k}(x)
\end{aligned}
$$

by the fact that $\lim _{n \rightarrow \infty} a_{n} D_{n}(x)=0$ if $x \neq 0$ where $D_{n}(x)=1 / 2+\cos x+\cos 2 x+$ $\ldots+\cos n x$.

Now applications of Abel's transformation and Lemma 1 and 2 yield,

$$
\begin{aligned}
& \int_{0}^{\pi}\left|\frac{a_{0}}{2}+\sum_{n=1}^{\infty} a_{n} \cos n x\right| d x \\
= & \int_{0}^{\pi}\left|\sum_{k=0}^{\infty} \Delta a_{k} D_{k}(x)\right| d x \\
= & \int_{0}^{\pi}\left|\sum_{k=0}^{\infty} A_{k} \frac{\Delta a_{k}}{A_{k}} D_{k}(x)\right| d x \\
\leq & \sum_{k=0}^{\infty}\left|\Delta A_{k}\right| \int_{0}^{\pi}\left|\sum_{j=0}^{k} \frac{\Delta a_{j}}{A_{j}} D_{j}(x)\right| d x \\
\leq & C \sum_{k=0}^{\infty}(k+1)\left|\Delta A_{k}\right|,
\end{aligned}
$$


since $\left|\frac{\Delta a_{i}}{A_{i}}\right|=\left|\propto_{i}\right| \leq 1$.

Then by Lemma 3 .

$$
\int_{0}^{\pi}\left|\frac{a_{0}}{2}+\sum_{n=1}^{\infty} a_{n} \cos n x\right|<\infty
$$

and satisfies the following inequality

$$
\int_{0}^{\pi} \cdot\left|\frac{a_{0}}{2}+\sum_{k=1}^{\infty} a_{k} \cos k x\right| \leq C \sum_{k=0}^{\infty}(k+1)\left|\Delta A_{k}\right| \leq C \sum_{k=0}^{\infty} A_{k} .
$$

This proves the theorem.

Proof of Theorem 2. Since $\Delta a_{n} \geq-\delta_{n}$, we have $\left|\Delta a_{n}\right| \leq a_{n}+2 \delta_{n}$. The convergence of the series $\Sigma_{k=1}^{\infty} k \delta_{k}<\infty$ implies that $\Sigma_{k=1}^{\infty} \delta_{k}<\infty$. Therefore, by using the condition that $a_{n} \rightarrow 0$, we have

$$
\sum_{n=1}^{\infty}\left|\Delta a_{n}\right| \leq \sum_{n=1}^{\infty} \Delta a_{n}+2 \sum_{n=1}^{\infty} \delta_{n}
$$

Thus, $\Sigma_{k=1}^{\infty} a_{n} \sin n x$ converges to $g(x)$ for every $x$.

We suppose that $a_{0}=0$ and $A_{0}=\max \left(\left|a_{1}\right|, A_{1}\right)$, we see that $A_{0} \leq \Sigma A_{k}$. Putting

$$
\begin{gathered}
\bar{D}_{0}(x)=\frac{-\cos x / 2}{2 \sin x / 2} \text { for } k \geq 1 \\
\bar{D}_{k}=\bar{D}_{0}(x)+\sin x+\sin 2 x+\ldots+\sin k x \\
=-\frac{\cos (k+1 / 2) x}{2 \sin x / 2}
\end{gathered}
$$

Then

$$
\begin{aligned}
& \int_{\pi / p+1}^{\pi}\left|\sum_{k=1}^{\infty} a_{k} \sin k x\right| d x \\
= & \int_{\pi / p+1}^{\pi}\left|\sum_{k=0}^{\infty} \Delta a_{k} D_{k}^{-}(x)\right| d x \\
= & \sum_{j=1}^{p} \int_{\pi / j+1}^{\pi / j}\left|\sum_{k=0}^{j-1} \Delta a_{k} D_{k}^{-}(x)\right| d x \\
& \quad+O\left(\sum_{j=1}^{p} \int_{\pi / j+1}^{\pi / j}\left|\sum_{k=j}^{\infty} \Delta a_{k} D_{k}^{-}(x)\right| d x\right) \quad I_{1}+I_{2}, \text { say }
\end{aligned}
$$

Application of Abel's transformation and lemma 2 yield:

$$
\begin{aligned}
\sum_{k=j}^{\infty} \Delta a_{k} D_{k}^{-}(x) & =\sum_{k=j}^{\infty} A_{k} \frac{\Delta a_{k}}{A_{k}} D_{k}^{-}(x) \\
& =\sum_{k=j}^{\infty} \Delta a_{k} \sum_{i=0}^{k} \frac{\Delta a_{i}}{A_{i}} D_{i}^{-}(x)-A_{j} \sum_{i=0}^{j-1} \frac{\Delta a_{i}}{A_{i}} D_{i}^{-}(x)
\end{aligned}
$$


Therefore

$$
\begin{aligned}
I_{2} \leq & \sum_{j=1}^{p} \int_{\pi / j+1}^{\pi / j}\left|\sum_{k=j}^{\infty} \Delta a_{k} D_{k}^{-}(x)\right| d x \\
\leq & \sum_{j=1}^{p}\left[\sum_{k=j}^{\infty}\left|\Delta A_{k}\right| \int_{\pi / j+1}^{\pi}\left|\sum_{i=0}^{k} \frac{\Delta a_{i}}{A_{i}} D_{i}^{-}(x)\right| d x\right. \\
& \left.+A_{j} \int_{\pi / j+1}^{\pi / j}\left|\sum_{i=0}^{j-1} \frac{\Delta a_{i}}{A_{i}} D_{i}^{-}(x)\right| d x\right] \\
\leq & \sum_{k=1}^{\infty}\left|\Delta A_{k}\right| \int_{\pi / j+1}^{\pi}\left|\sum_{i=0}^{k} \frac{\Delta a_{i}}{A_{i}} D_{i}^{-}(x)\right| d x \\
& +C \sum_{j=1}^{\infty} A_{j} \int_{\pi / j+1}^{\pi / j} \frac{j \sin x / 2}{2 \sin x} \\
\leq & C \sum_{k=1}^{\infty}(k+1)\left|\Delta A_{k}\right|+C \sum_{j=1}^{\infty} A_{j}, \text { by Lemma } 1, \\
\leq & C \sum_{k=1}^{\infty}(k+1)\left|\Delta A_{k}\right|,
\end{aligned}
$$

Hence by Lemma 3

$$
I_{2} \leq C \sum_{k=1}^{\infty}(k+1)\left|\Delta A_{k}\right|<\infty .
$$

For all $x \in[0, \pi] k=0,1,2, \cdots$

$$
D_{k}^{-}(x)=-\frac{1}{x}+O(k+1) .
$$

we have

$$
\begin{aligned}
I_{1} & =\sum_{j=1}^{p} \int_{\pi / j+1}^{\pi / j}\left|\sum_{k=0}^{j-1} \Delta a_{k} D_{k}^{-}(x)\right| d x \\
& =\sum_{j=1}^{p} \int_{\pi / j+1}^{\pi / j}\left|\sum_{k=0}^{j-1} \Delta a_{k}\right| \frac{d x}{x}+O\left(\sum_{j=1}^{p} \int_{\pi / j+1}^{\pi / j} \sum_{k=0}^{j-1}\left|\Delta a_{k}\right|(k+1) d x\right) \\
& =\sum_{j=1}^{p} \frac{\left|a_{j}\right|}{j}+O\left(\sum_{j=1}^{p} \frac{\left|a_{j}\right|}{j^{2}}\right)+O\left(\sum_{j=1}^{p} \sum_{k=0}^{j-1} \frac{(k+1)\left|\Delta a_{k}\right|}{j^{2}}\right)
\end{aligned}
$$

But

$$
\sum_{j=1}^{p} \frac{\left|a_{j}\right|}{j^{2}} \leq C \max _{j}\left|a_{j}\right| \leq C \sum_{k=1}^{\infty}\left|\Delta a_{k}\right| \leq C \sum_{k=1}^{\infty} A_{k}
$$


and

$$
\sum_{j=1}^{p} \sum_{k=0}^{j-1} \frac{(k+1)}{j^{2}}\left|\Delta a_{k}\right| \leq C \Sigma_{k=0}^{\infty}\left|\Delta a_{k}\right| \leq C \sum_{k=1}^{\infty} A_{k} .
$$

Therefore,

$$
I_{1}=\sum_{j=1}^{p} \frac{\left|a_{j}\right|}{j}+O\left(\sum_{k=1}^{\infty} A_{k}\right)
$$

Hence

$$
\int_{\pi / p+1}^{\pi}\left|\sum_{k=1}^{\infty} a_{k} \sin k x\right| d x=\sum_{k=1}^{p} \frac{\left|a_{k}\right|}{k}+O\left(\sum_{k=1}^{\infty} A_{k}\right)
$$

This proves Theorem 2.

\section{References}

[1] Boas, R.P., "Quasi-positive sequence and trigonometric series", Proc. Lond. Math. Soc. 14A(1965), 38-48.

[2] Fomine, G.A., "On linear method for summing Fouries series", Mat. Sb. 66 (107), (1964), 114-152.

[3] Kolmogorov, A.H., "Sur $\ell^{\prime}$ order de grandeur des coefficients de la series de Fourier-Lebesgue", Bull. Acad. Polon. Sci (A). Sci. Math. (1923), 83-86.

[4] Sidon, S., "Hinreichende Bedingungen Fur den Fourier-Charakter ainer trigonometrischen Reihe", J. London Math. Soc. 14(1939), 158-160.

[5] Sing, N. and Sharma, K.M., "Integrability of trigonometric series", Journal of the Indian Math. Soc. 49 (1985), 31-38.

[6] Szasz, O., "Quasi-monotone series", Amer. J. Math. 70(1948), 203-206.

[7] Teljakoveskii, S.A., "Concerning a sufficient condition of Sidon for the integrability of trigonometric series", Math. Notes (1973), 742-748.

[8] Young, W.H., "On the Fourier series of bounded functions", Proc. London, Math. Soc. (2) (1913), 41-70.

Department of Mathematics, Aligarh Muslim University, Aligarh, India. 